\title{
Novel Method of Sampling the Gastrointestinal Muscle Layer: Feasibility of Endoscopic Muscular Resection with a Ligation Device in an in Vivo Porcine Model
}

\author{
Osamu Goto, Kazutoshi Higuchi, Atsushi Tatsuguchi, \\ Eriko Koizumi, Hiroto Noda, Takeshi Onda, \\ Jun Omori, Mitsuru Kaise and Katsuhiko Iwakiri \\ Department of Gastroenterology and Hepatology, Nippon Medical School Hospital, Tokyo, Japan
}

Background: Gastrointestinal muscular sampling is useful in the histological assessment of functional gastrointestinal disorders. We devised a new sampling method to obtain a large volume of muscle tissues and then investigated the feasibility and safety of endoscopic muscular resection with a ligation device in an in vivo porcine model.

Methods: After establishing a submucosal tunnel, a rubber band was placed on the muscle tissue by sucking the exposed muscle layer. Thereafter, the established pseudopolyp was removed using an electrocautery snare, and the entry site of the submucosal tunnel was closed endoscopically. This procedure was performed at three sites in the esophagus and stomach of two pigs. The technical success, histology, and survival rate on postoperative day 7 were examined postoperatively.

Results: We successfully completed the mentioned procedure in 11 of the 12 sites (92\%), without the occurrence of severe adverse events. The median diameters of obtained tissues from the esophagus and stomach, respectively, were $5 \mathrm{~mm}$ and $10 \mathrm{~mm}$. Histologically, both the inner and outer muscle layers were included in all specimens. The postprocedural course was found uneventful in both pigs during the observatory period.

Conclusions: Endoscopic muscular resection using a ligation device enabled us to obtain large and thick muscle tissue samples. This approach may facilitate more precise histological assessments of functional gastrointestinal disorders. (J Nippon Med Sch 2021; 88: 441-447)

Key words: endoscopic muscle biopsy, submucosal endoscopy, functional disorder

\section{Introduction}

Gastrointestinal motor dysfunction has shown various phenotypes among individual organs ${ }^{1-3}$. Several possible causes of this phenomenon have been proposed, including autoimmune disease, infection, and genetic disease; however, the definite etiology remains to be unknown ${ }^{4-6}$. To date, a variety of multidirectional approaches have been introduced, among which pathological assessments are important to elucidate the mechanism of this condition.

Gastrointestinal muscular sampling is a helpful technique to investigate the histology and pathogenesis of functional gastrointestinal disorders. In typical primary esophageal achalasia, the degeneration of the myenteric plexus and reduction of the interstitial cells of Cajal (ICC) are observed in the esophageal muscle layer and are collectively considered as a cause of impaired relaxation in the lower esophageal sphincter ${ }^{7-11}$. Similarly, it is thought that nerve damage contributes to delayed gastric emptying, with various resulting unpleasant symptoms in gastroparesis ${ }^{12,13}$ and severe constipation ${ }^{14}$.

Full-thickness sampling of the gut wall is considered an ideal means to ensure a complete pathological evaluation. However, this technique is generally accompanied

Correspondence to Osamu Goto, MD, PhD, Department of Gastroenterology and Hepatology, Nippon Medical School Hospital, 1-1-5 Sendagi, Bunkyo-ku, Tokyo 113-8603, Japan

E-mail: o-goto@nms.ac.jp

https://doi.org/10.1272/jnms.JNMS.2021_88-602

Journal Website (https://www.nms.ac.jp/sh/jnms/) 

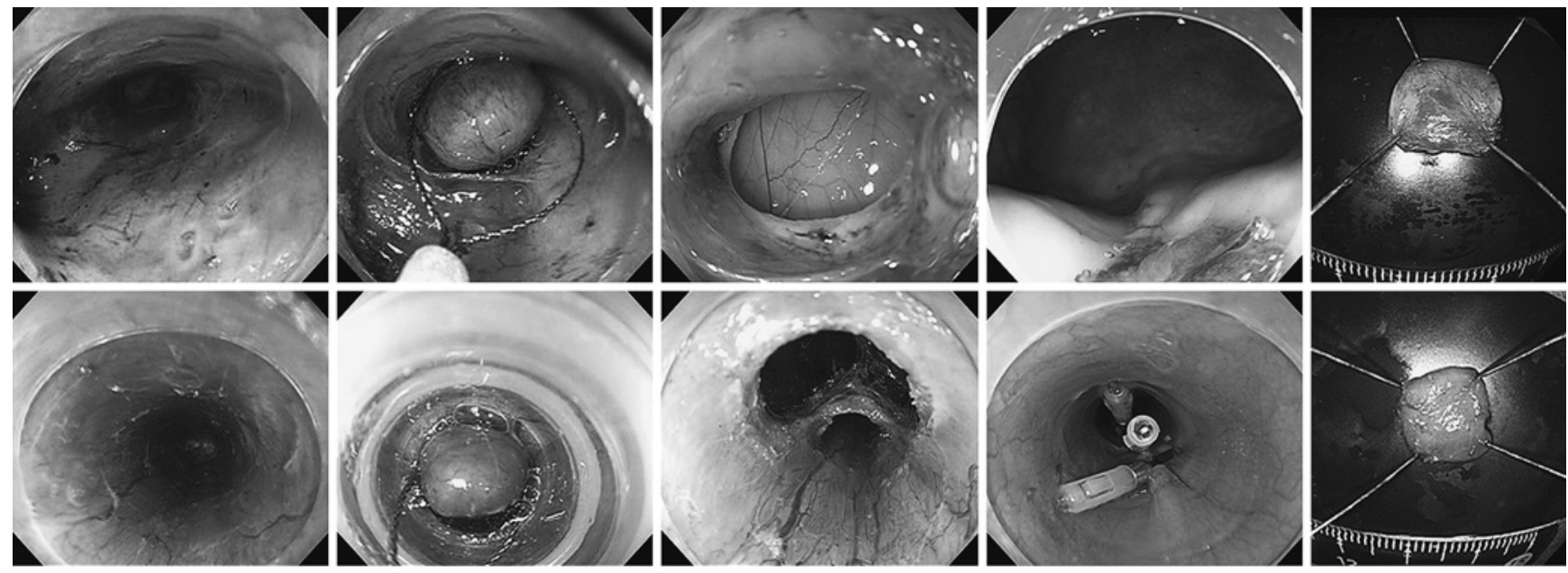

$$
\begin{array}{c|c|c|c|c}
\mathrm{a} & \mathrm{b} & \mathrm{c} & \mathrm{d} & \mathrm{e} \\
\hline \mathrm{f} & \mathrm{g} & \mathrm{h} & \mathrm{i} & \mathrm{j}
\end{array}
$$

Fig. 1 Endoscopic muscular resection using a ligation device

a A submucosal tunnel is created in the stomach. $\mathbf{b}$ After ligating the exposed muscle layers with the rubber band, the created pseudopolyp was removed using an electrocautery snare. c An abdominal space can be seen through the muscular defect. $\mathbf{d}$ The entry site was closed using an endoscopic hand-suturing technique. e The obtained muscle tissue from the stomach can be seen. $\mathbf{f}$ The same procedure was applied to the esophagus. $\mathrm{g}$ The submucosal tunnel was determined to be thinner in the esophagus than in the stomach. $\mathbf{h}$ A mediastinal space can be observed. $\mathbf{i}$ The tunnel entrance was clipped. $\mathbf{j}$ The obtained muscle tissue from the esophagus.

by a surgical approach for end-stage dysmotility or other disease such as gastrointestinal cancer $^{7-14}$, and surgery aiming to obtain full-thickness tissue may be too invasive in some cases. In this context, endoscopic full-thickness sampling offers promise as an alternative to using a surgical approach, which requires secure closure for a transluminal defect ${ }^{15,16}$.

Endoscopic muscle biopsy is a less invasive method for obtaining muscle tissue. Particularly, taking biopsies in a submucosal tunnel can be safely completed using the peroral endoscopic myotomy (POEM) technique ${ }^{5,17-19}$. However, the outcomes of the subsequent histological assessments may be deemed unsatisfactory because the muscular tissues obtained with biopsy forceps are often too small to precisely evaluate the density of inflammatory cells, the decline of the myenteric plexus, the concentration of ICCs, and degenerative changes in the tissues. Furthermore, pathologists may be unable to discriminate between inner and outer muscle layers due to the small volume of the sampled tissues, so completing accurate assessments can sometimes be difficult.

We have devised a new sampling method to obtain a large volume of muscle tissues involving an endoscopic mucosal resection technique with a ligation device. In this study, we investigated the feasibility and safety profiles of this method in an in vivo porcine model.

\section{Materials and Methods}

\section{Design and Settings}

After approval was secured from the institutional review board for animal ethics [approval no. 19009-(0)], a survival study was performed using two live female pigs in whom we performed endoscopic muscular resection in the esophagus and stomach. The two pigs, which weighed $19.5 \mathrm{~kg}$ and $19.8 \mathrm{~kg}$, respectively, underwent the procedure in question under general anesthesia with endotracheal intubation.

\section{Endoscopic Muscular Resection}

One endoscopist who has performed more than 800 and 15 cases of endoscopic submucosal dissection and POEM, respectively, conducted the procedures in this study. A flexible endoscope was inserted perorally through an overtube. After rinsing the inside of the upper gastrointestinal tract and aspirating the fluid in the esophagus and the stomach, a submucosal tunnel was created in the stomach using submucosal injection, mucosal incision, and linear submucosal dissection from the upper side on the posterior wall to the direction of the greater curvature with the POEM technique (Fig. 1a) ${ }^{20}$. After creating a tunnel measuring approximately $8 \mathrm{~cm}$ in length, a rubber band (70420002; Sumitomo Bakelite, Co., Ltd., Tokyo, Japan), generally used for esophageal variceal ligation, was then placed on the distal side of 
the exposed muscle layer in the tunnel by sucking the muscle tissue to create a pseudopolyp (Fig. 1b). Subsequently, the pseudopolyp was removed using an electrocautery snare measuring $10 \mathrm{~mm}$ in diameter (SD-210 L10; Olympus Co., Ltd., Tokyo, Japan) (Fig. 1c). If the ligation failed after several trials due to slippage of the band prior to snare resection, the completion of muscular resection was assisted by partial muscular incision with a needle-type knife (KD-655 L; Olympus). The removed muscle specimen and the rubber band were transorally retrieved by withdrawal of the endoscope with suction or by using grasping forceps. After three consecutive sessions of resection from the distal to the proximal side, the entry site of the submucosal tunnel was closed by endoscopic hand-suturing (EHS) (Fig. 1d ${ }^{21}$. The retrieved specimen was pinned on the rubber board and further sank in 10\% neutral buffered formalin (Fig. 1e). Subsequently, the same procedure was conducted in the esophagus (Fig. 1f $\sim \mathbf{1 j}$ ). Endoclips (HX-610-090 L; Olympus) were used for the closure of the esophageal submucosal tunnel due to the narrow lumen (Fig. 1i). When intraoperative bleeding occurred, hemostasis was attempted using the tip of the snare or hemostatic forceps (FD-410LR; Olympus). The whole endoscopic procedure was recorded on video.

This procedure was performed in the 2 pigs in order to yield 12 sites in total (6 each in the esophagus and the stomach). A liquid diet and regular food were provided on postoperative days (PODs) 1 and 2, respectively. Following endoscopic observation and subsequent euthanasia of both animals on POD 7, autopsies are then performed.

\section{Outcome Measures}

As a technical outcome, we first assessed the feasibility of the mentioned technique. Completeness, the number of re-ligations, the procedural duration (including for submucosal tunnel creation, muscular resection in the three sites, specimen retrieval, and entry site closure), the specimen size, and the occurrence of any intraoperative adverse events including bleeding were examined by watching the recorded video. Separately, to investigate the safety of the procedure, survival at 1 week was evaluated.

Meanwhile, to evaluate the usefulness of this technique, we assessed the histology of the specimen. The resected muscle tissues and the tissues surrounding the muscular defects in the isolated esophagus and stomach were fixed in $10 \%$ neutral buffered formalin immediately after their retrieval during the procedure and autopsy, re- spectively. These specimens were halved into two pieces; embedded with paraffin; sliced into $4 \mu \mathrm{m}$ sections; and stained with hematoxylin and eosin, anti-desmin antibody (ab8976; 1:100 dilution; Abcam plc, Cambridge, UK), anti-S100 alpha antibody (ab11428, 1:2500 dilution; Abcam), and anti-c-Kit antibody (ab178527, 1:100 dilution; Abcam). Using the resected muscle tissues, we examined whether the tissue has included both the inner and outer muscle layers and if the direction of the specimens could be determined. In the surrounding tissues of the muscular defect, histological changes in the defect were assessed. Furthermore, the number of myenteric plexi and the density of ICCs [total number in five highpower fields (HPFs)] were evaluated in the muscular specimens.

\section{Statistics}

Because this experiment was a preclinical pilot study designed to investigate the feasibility and usefulness of the new endoscopic technique, a sample size could not be calculated. We used a mean time for the procedural duration of muscular resection per lesion and a median with a range for the specimen size, the number of myenteric plexi, and the density of ICCs.

\section{Results}

\section{Technical Feasibility}

We were able to complete endoscopic muscular resection using a ligation device at 11 sites (5 esophageal sites and 6 gastric sites; $92 \%$ of total sites) without the occurrence of severe adverse events. At the five sites (four in the esophagus and one in the stomach), re-ligation was deemed necessary. In one of the four esophageal sites, band ligation was abandoned and part of the muscle tissue was finally removed by the assistance of the needletype knife. Intraprocedural bleeding was recorded at two sites (one in the esophagus and one in the stomach), which was endoscopically stopped with the use of hemostatic forceps. At one stomach site, a rubber band was broken into two pieces by snaring with the muscle tissue and energization. In this case, all of the pieces were retrieved endoscopically following the retrieval of the specimen.

The procedural durations were 52 minutes for the stomach and 57 minutes for the esophagus in the first pig and 87 minutes for the stomach and 36 minutes for the esophagus in the second pig, respectively. The procedures were prolonged due to ligation failure and subsequent hybrid resection in the esophagus of the first pig and due to band fracture and bleeding in the stomach of 

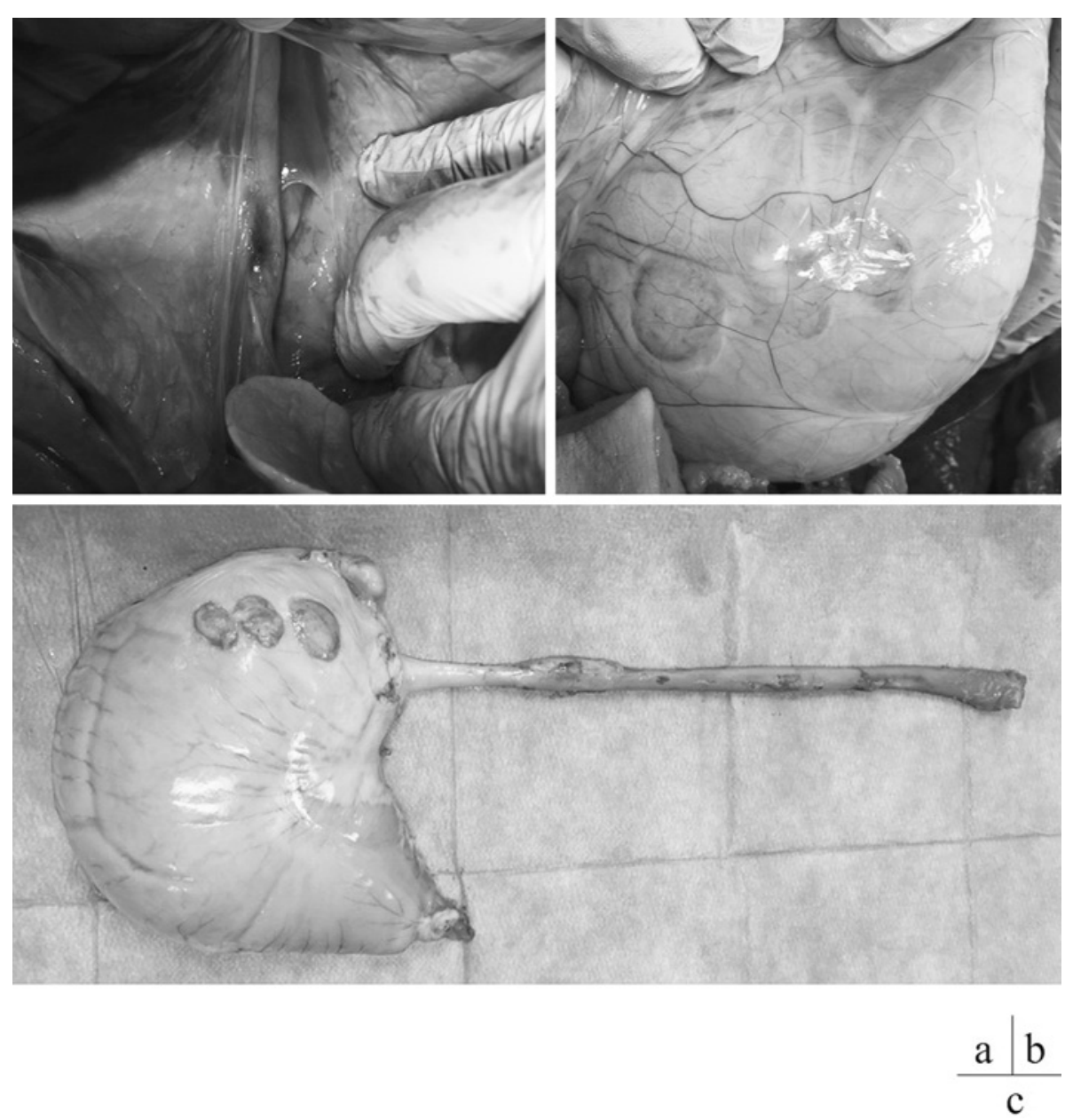

Fig. 2 Obtained specimens and autopsy results on postoperative day 7 a The muscular defect site in the esophagus is presented but no abscess is seen. $\mathbf{b}$ In the stomach, covering of the muscular defects with the omentum is observed. c Both organs were well preserved without severe injury.

the second pig, respectively. The mean durations required for endoscopic muscular resection per lesion, which involved band ligation, snare resection, retrieval, and hemostasis (if performed), were 11 minutes in the stomach and 7 minutes in the esophagus, respectively.

The median maximum diameter of the obtained muscle tissues was 10 (range: 7-12) $\mathrm{mm}$ in the stomach and 5 (range, 4-7) $\mathrm{mm}$ in the esophagus, respectively. The postprocedural course was determined to be uneventful in both pigs. Neither evidence of perforation nor diverticular formation was observed endoscopically on POD 7.

\section{Autopsy and Histology}

During the autopsy, we saw mild tissue adhesions surrounding the esophageal sites (Fig. 2a). In the gastric sites, the seromuscular defects were covered with the greater omentum (Fig. 2b). No instance of apparent abscess formation or severe inflammation was noted (Fig. 2 c).

Histologically, the two muscular layers were included in all specimens (Fig. $\mathbf{3 a}$ and $\mathbf{3 b}$ ). By confirming the location of the partially remaining submucosal tissue with thermal damage or the existence of serosa in the stomach, the direction at the radial axis (inner or outer) of the obtained specimens could be determined, whereas the direction at the long axis (oral or anal) as well as at the short axis (left or right) remained to be undetermined. At the resection sites, undamaged healthy mucosa covering the muscular defects was observed (Fig. 3c and 3d).

In the muscle specimens stained by anti-S100 alpha antibody, a quantitative assessment of myenteric plexus was performed. The median values of the maximal number of myenteric plexi were 7.5 (range: $4-18$ ) in the stomach and 6 (range: 1-7) in the esophagus, respectively. During immunohistochemistry analysis using the anti-cKit antibody, the mean value of the density of ICCs per HPF was determined to be 8 (range: 3-12) in the stomach and 4 (range: $0-30$ ) in the esophagus, respectively. Representative microscopic images of the histology are pre- 

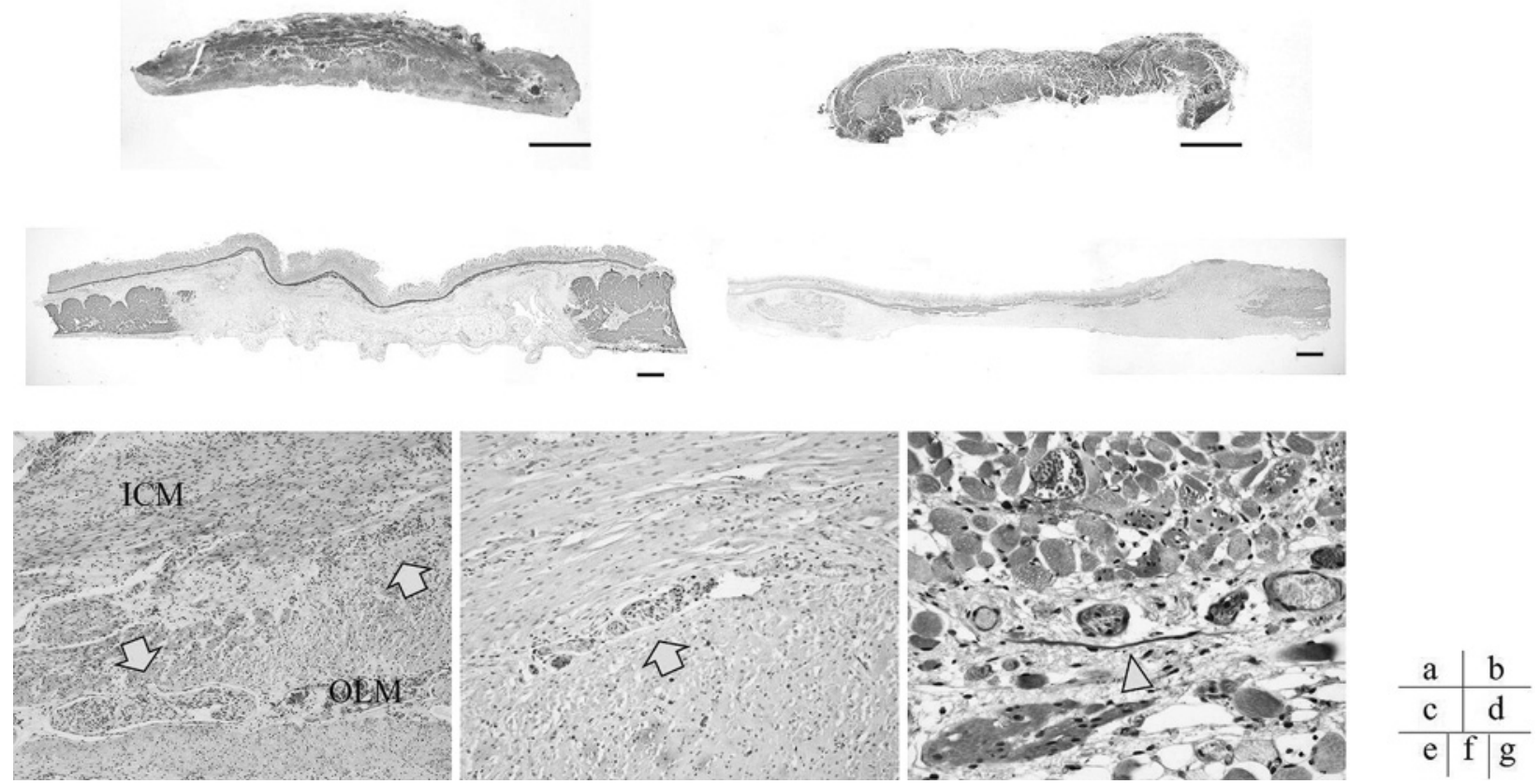

Fig. 3 Histology of removed muscle tissues and surroundings

The muscle specimens from the stomach (a) and the esophagus (b). In both specimens, inner circular and outer longitudinal muscle layers are observed with a right radial direction $(\mathrm{bar}=1 \mathrm{~mm})$. The surrounding tissue from the stomach $(\mathbf{c})$ and the esophagus (d) stained with the anti-desmin antibody. The muscular defects were covered with undamaged normal mucosa (bar $=1 \mathrm{~mm}$ ). e Hematoxylin and eosin staining of the muscle specimen in the stomach $(\times 100)$. An example of myenteric plexus (arrow) is seen in the muscle layers. ICM, inner circular muscle; OLM, outer longitudinal muscle. f A myenteric plexus (arrow) stained with the anti-S100 alpha antibody $(\times 200)$. g ICCs (arrowhead) were stained with the anti-c-Kit antibody $(\times 400)$.

sented in (Fig. 3e-g).

\section{Discussion}

In this preclinical experiment, we have successfully proved that endoscopic muscular resection using a ligation device is feasible, safe, and potentially useful for the histological evaluation of gastrointestinal functional disorders. By adopting this technique, whole muscle layers can be sampled less invasively using easily accessible endoscopic devices.

This approach ensures the collection of a large amount of gastrointestinal muscle tissue in a layered fashion. By obtaining a certain volume of muscular tissue, evaluating the existence of inflammatory cell infiltration, degeneration, or fibrosis in the muscle tissue has now been made possible as well as discerning the density of intramuscular myenteric plexi and ICCs, which are sometimes difficult to assess in small tissue samples taken using biopsy forceps ${ }^{17}$. Particularly in the case of a functional disorder such as esophageal achalasia or gastroparesis, we cannot determine whether the absence of myenteric plexus or ICCs is due to a progression of the disease or a simple sampling error because these do not exist so abundantly as to be measured easily. Furthermore, our described technique tells us the radial direction of the muscle layer. By pinning the specimen down in a round disk shape, fixing it, and cutting it vertically to the disk surface, we can evaluate the cross-sectional histology of two muscle layers and intramuscular tissue, which may not be available when evaluating a small specimen secured by biopsy forceps due to the uncertainty of the direction and thus undesirable slicing of the specimen. Accordingly, owing to this technique, we can obtain 5 to $10 \mathrm{~mm}$ prepared slides showing cross-sectional double muscle layers, which are sufficient in performing histological analyses in terms of both volume and direction. Regarding the amount of tissue, a direct comparison of the specimen between muscle biopsy and this technique might be ideal. However, we can easily estimate the volume of tissue obtainable by biopsy forceps because it is subject to the size of the forceps' cup and not so variable $(2-3 \mathrm{~mm}$ in size).

Technically, the feasibility of this procedure appears to be acceptable, although one week of the observatory period may be too short to assess late-onset adverse events e.g., abscess, adhesion or diverticular formation. In this 
study, we sutured the entry site of the gastric submucosal tunnel by the EHS technique, which was still hardly accessible. The submucosal tunnel may be closed according to the endoscopist's preferred technique e.g., the use of Over-the-scope $\mathrm{clip}^{15}$, providing a secure and persistent closure is obtained. In terms of the avoidance of adverse events, the muscular defect should be closed. However, the muscular defect closure in the narrow submucosal tunnel is far difficult and almost impossible. If it is doable, we have to consider a potential risk of postoperative stricture in the suturing site, particularly in the esophagus. Therefore, we guess that it would be favorable that the muscular defect remains open to avoid more relevant risks.

This technique is also expected to be applicable for deployment to evaluate the histology of the rectum in cases showing chronic constipation, although we did not try to confirm this because of the difficulty of preprocedural preparation in pigs in performing this assessment. We experienced several cases requiring re-ligation, particularly in the esophagus, including one case marked by a failure to snare. One possible reason for this may be due to the thin esophagus of young pigs and the small diameter of the submucosal tunnel in this investigation. By creating a broader submucosal tunnel, we might be able to obtain a working space that is sufficient for long-lasting ligation which promotes successful snaring. However, in clinical settings (e.g., jackhammer esophagus), the muscle layers sometimes become hypertrophic, which may hamper the creation of a pseudopolyp. The clinical durability of this technique should be investigated in various types of motor dysfunction.

To evaluate the suitability of this technique in humans, a clinical study must be conducted in the future. Although intraoperative bleeding will be managed endoscopically as we have experienced in this study, particularly in the stomach, areas where large vessels are penetrated (e.g., lesser-anterior and lesser-posterior areas in the gastric body) should not be selected. Furthermore, rubber band fracture is an undesirable event during pseudopolyp resection. Because the rubber band is a foreign material, all fragments should be completely retrieved in case of a fracture. Otherwise, endoscopic mucosal resection with a cap-fitted technique or other endoscopic mucosal resection technique may be useful and safe to adopt in human cases.

Performing endoscopic muscle biopsy by using biopsy forceps is considered to be simple and safe when compared to the described technique $e^{5,17-19}$ and can obtain mus- cle tissue in a double-layered fashion if it is sufficiently thin. However, the volume of the obtained sample may sometimes be too small to assess the histology, and the right direction of muscle layers can be difficult to determine as described above. Conducting endoscopic fullthickness resection by using the "suture-and-cut" technique with an over-the-scope clip and snaring approach is another possibility ${ }^{15,16}$. This technique is also a simple and useful means by which to examine the histology of the gut wall, although a special and costly device is required. Furthermore, performing a full-thickness evaluation is not always mandatory because mucosal and submucosal tissue can be easily and safely obtained using conventional mucosal biopsy.

This present study has several limitations. First, this was an animal study conducted using healthy live pigs with a short survival period. To confirm that this technique is feasible and safe for adoption into clinical practice, a human pilot study will be designed involving patients with gastrointestinal functional disorders. Second, the sample size was small ( $n=2$ pigs), although we believe that the statistical evaluation is not significantly important due to the characteristics of this study and given the aim of investigating the feasibility of this technique was achieved. Third, the same therapeutic endoscopist performed all procedures; therefore, the generalizability of this technique across different centers and levels of operator experience remains unknown.

In conclusion, this animal survival study demonstrated that endoscopic muscular resection with a ligation device is feasible and safe and should considered for possible introduction in clinical practice. This technique provided us with cross-sectional histological images of large and thick gastrointestinal muscle layers and therefore is useful for facilitating accurate pathological assessments of functional disorders.

Acknowledgements: We thank Mrs. Akiko Takeda for technical assistance with the tissue staining.

Funding: This study was partly supported by a Grant-in-aid for Scientific Research (C) from the Ministry of Education, Culture, Sports, Science and Technology in Japan awarded from 2018 to 2020 (grant no. 18K07919).

Conflict of Interest: The authors have no conflicts of interest.

References

1. Rohof WOA, Bredenoord AJ. Chicago classification of 
esophageal motility disorders: Lessons learned. Curr Gastroenterol Rep. 2017;19:37.

2. Grover M, Farrugia G, Stanghellini V. Gastroparesis: a turning point in understanding and treatment. Gut. 2019; 68:2238-50.

3. Bharucha AE, Pemberton JH, Locke GR 3rd. American Gastroenterological Association technical review on constipation. Gastroenterology. 2013;144:218-38.

4. Ghoshal UC, Daschakraborty SB, Singh R. Pathogenesis of achalasia cardia. World J Gastroenterol. 2012;18:3050-7.

5. Ikebuchi $\mathrm{Y}, \mathrm{Kanda} \mathrm{T}$, Ikeda $\mathrm{H}$, et al. Identification of human herpes virus 1 encoded microRNAs in biopsy samples of lower esophageal sphincter muscle during peroral endoscopic myotomy for esophageal achalasia. Dig Endosc. 2020;32:136-42.

6. Sharma A, Coles M, Parkman H, et al. Gastroparesis in the 2020s: New Treatments, New Paradigms. Curr Gastroenterol Rep. 2020;22:23.

7. Goldblum JR, Whyte RI, Orringer MB, et al. Achalasia. A morphologic study of 42 resected specimens. Am J Surg Pathol. 1994;18:327-37.

8. Goldblum JR, Rice TW, Richter JE. Histopathologic features in esophagomyotomy specimens from patients with achalasia. Gastroenterology. 1996 Sep;111(3):648-54.

9. Raymond L, Lach B, Shamji FM. Inflammatory aetiology of primary oesophageal achalasia: an immunohistochemical and ultrastructural study of Auerbach's plexus. Histopathology. 1999;35:445-53.

10. Hoshino M, Omura N, Yano F, et al. Immunohistochemical study of the muscularis externa of the esophagus in achalasia patients. Dis Esophagus. 2013;26:14-21.

11. Sodikoff JB, Lo AA, Shetuni BB, et al. Histopathologic patterns among achalasia subtypes. Neurogastroenterol Motil. 2016;28:139-45.

12. Iwasaki H, Kajimura M, Osawa S, et al. A deficiency of gastric interstitial cells of Cajal accompanied by decreased expression of neuronal nitric oxide synthase and substance $\mathrm{P}$ in patients with type 2 diabetes mellitus. J Gastroenterol. 2006;41:1076-87.

13. Lin Z, Sarosiek I, Forster J, et al. Association of the status of interstitial cells of Cajal and electrogastrogram parameters, gastric emptying and symptoms in patients with gastroparesis. Neurogastroenterol Motil. 2010;22:56-61, e 10.

14. Lee JI, Park H, Kamm MA, et al. Decreased density of interstitial cells of Cajal and neuronal cells in patients with slow-transit constipation and acquired megacolon. J Gastroenterol Hepatol. 2005;20:1292-8.

15. Ngamruengphong S, Thompson E, McKnight M, et al. Endoscopic full-thickness muscle biopsy for rectal tissue sampling in patients with severe gut motility disorders: an initial experience (with video). Gastrointest Endosc. 2019;89:1242-7.

16. Rajan E, Gostout CJ, Wong Kee Song LM, et al. Innovative gastric endoscopic muscle biopsy to identify all cell types, including myenteric neurons and interstitial cells of Cajal in patients with idiopathic gastroparesis: a feasibility study (with video). Gastrointest Endosc. 2016;84:512-7.

17. Nakajima $N$, Sato $H$, Takahashi $K$, et al. Muscle layer histopathology and manometry pattern of primary esophageal motility disorders including achalasia. Neurogastroenterol Motil. 2017;29.

18. Sato H, Nakajima N, Hasegawa G, et al. Immunohistochemical differentiation of eosinophilic esophageal myositis from eosinophilic esophagitis. J Gastroenterol Hepatol. 2017;32:106-13.

19. Sato H, Takahashi K, Mizuno KI, et al. Esophageal motility disorders: new perspectives from high-resolution manometry and histopathology. J Gastroenterol. 2018;53:48493.

20. Inoue $\mathrm{H}, \mathrm{Minami} \mathrm{H}, \mathrm{Kobayashi} \mathrm{Y}$, et al. Peroral endoscopic myotomy (POEM) for esophageal achalasia. Endoscopy. 2010;42:265-71.

21. Goto O, Oyama T, Ono $\mathrm{H}$, et al. Endoscopic handsuturing is feasible, safe, and may reduce bleeding risk after gastric endoscopic submucosal dissection: a multicenter pilot study (with video). Gastrointest Endosc. 2020; 91:1195-202.

(Received, October 30, 2020)

(Accepted, December 11, 2020)

Journal of Nippon Medical School has adopted the Creative Commons Attribution-NonCommercial-NoDerivatives 4.0 International License (https://creativecommons.org/licenses/by-nc-nd/4.0/) for this article. The Medical Association of Nippon Medical School remains the copyright holder of all articles. Anyone may download, reuse, copy, reprint, or distribute articles for non-profit purposes under this license, on condition that the authors of the articles are properly credited. 